\title{
Eficiência de Herbicidas para a Cultura do FeiJão-Caupi ${ }^{1}$
}

\author{
Efficiency of Herbicides for Cowpea Crop
}

\author{
SILVA, K.S. ${ }^{2}$, FREITAS, F.C.L. ${ }^{2}$, SILVEIRA L.M. ${ }^{2}$, LINHARES, C.S. ${ }^{2}$, CARVALHO, D.R. ${ }^{2}$ e \\ LIMA, M.F.P. ${ }^{2}$
}

\begin{abstract}
RESUMO - Com o objetivo de avaliar a seletividade e eficácia de herbicidas para a cultura do feijão-caupi (Vigna unguiculata), conduziu-se um experimento no esquema de parcelas subdivididas, no delineamento de blocos casualizados. Nas parcelas, foram avaliados nove herbicidas/misturas (S-metolachlor, bentazon + imazamox, S-metolachlor + bentazon + imazamox, imazamox + fluazifop-p-butyl, imazethapyr + fluazifop-p-butyl, bentazon + fluazifopp-butyl, bentazon + imazamox + fluazifop-p-butyl, lactofen + fluazifop-p-butyl e fluazifop-pbutyl) e uma testemunha sem herbicidas, e nas subparcelas foram avaliados dois tratamentos: com capinas e sem capinas, para determinar a seletividade e a eficácia dos herbicidas, respectivamente. Os herbicidas S-metolachlor, bentazon + imazamox, S-metolachlor + bentazon + imazamox, imazamox + fluazifop-p-butyl, imazethapyr + fluazifop-p-butyl, bentazon + fluazifop-p-butyl, bentazon + imazamox + fluazifop-p-butyl e fluazifop-p-butyl foram seletivos para a cultura. A mistura lactofen + fluazifop-p-butyl causou severa intoxicação no feijãocaupi, com posterior recuperação e produtividade igual ou superior à dos demais tratamentos. As principais espécies de plantas infestantes foram: Cleome affinis, Trianthema portulacastrum, Amaranthus spinosus, Commelina benghalensis e Digitaria bicornis, sendo todas controladas com eficiência pelos herbicidas S-metolachlor aplicado em pré-emergência + bentazon + imazamox aplicados em pós-emergência e lactofen + fluazifop-p-butyl aplicados em pós-emergência. Conclui-se que a eficiência de herbicidas para o feijão-caupi depende da seletividade para a cultura, bem como de sua eficácia no controle de plantas daninhas, a qual pode variar de acordo com a comunidade infestante.
\end{abstract}

Palavras-chave: Vigna unguiculata, controle químico, planta daninha, seletividade.

\begin{abstract}
In order to evaluate the selectivity and efficiency of herbicides for the crop of cowpea (Vigna unguiculata), an experiment was conducted in split plots in a randomized complete block design. The plots consisted of nine herbicides/mixtures (S-metolachlor, bentazon + imazamox, Smetolachlor + bentazon + imazamox, imazamox + fluazifop-p-butyl, imazethapyr + fluazifop-p-butyl, bentazon + fluazifop-p-butyl, bentazon + imazamox + fluazifop-p-butyl, lactofen + fluazifop-p-butyl, fluazifop-p-butyl) and a control without herbicides, while the subplots consisted of the evaluation of two treatments : with weeding and without weeding, to determine the selectivity and effectiveness of the herbicides, respectively. The herbicides $S$-metolachlor, imazamox + bentazon, bentazon $+S-$ metolachlor + imazamox, imazamox + fluazifop-p-butyl, imazethapyr + fluazifop-p-butyl, bentazon + fluazifop-p-butyl, imazamox + bentazon + fluazifop-p-butyl and fluazifop-p-butyl were selective for the cowpea crop. The mixture lactofen + fluazifop-p-butyl caused severe intoxication in the cowpea crop with subsequent recovery and yield equal to or higher than the other treatments. The main weed species were: Cleome affinis, Trianthema portulacastrum, Amaranthus spinosus, Commelina benghalensis and Digitaria bicornis, all being efficiently controlled by the herbicide S-metolachlor applied at pre-emergence + imazamox + bentazon applied at post-emergence and lactofen + fluazifop-p-butyl applied at post-emergence. It was concluded that the efficiency of the herbicides for cowpea depends on the selectivity for the crop, as well as on their effectiveness in controlling weeds, which may vary according to the weed community.
\end{abstract}

Keywords: Vigna unguiculata, chemical control, weed, selectivity.

Recebido para publicação em 20.9.2013 e aprovado em 20.1.2014.

Universidade Federal Rural do Semi-Árido, Mossoró-RN, Brasil, <kaliane_kaka@hotmail.com>.

Planta Daninha, Viçosa-MG, v. 32, n. 1, p. 197-205, 2014 


\section{INTRODUÇÃO}

O feijão-caupi (Vigna unguiculata) é uma das leguminosas mais consumidas e cultivadas no Brasil, especialmente nas regiões Norte e Nordeste. No entanto, nos últimos anos, tem-se observado grande expansão da área cultivada também na região CentroOeste, onde é incorporado aos arranjos produtivos como safrinha após as culturas da soja e do arroz, e, em alguns locais, como cultura principal (Freire Filho et al., 2011). Entre os problemas que afetam a produtividade da cultura, destaca-se a interferência das plantas daninhas, que, quando não controladas, podem reduzir o rendimento de grãos em até 90\% (Freitas et al., 2009a).

A estratégia de controle das plantas daninhas mais utilizada no cultivo do feijãocaupi é a capina manual, por se tratar de uma cultura explorada principalmente no sistema de agricultura familiar (Freitas et al., 2009b). Entretanto, em áreas extensas, o alto custo de mão de obra e a dificuldade de encontrar operários em quantidade suficiente tornam esse método apenas complementar (Silva \& Silva, 2007). Já a capina mecanizada possui bom rendimento operacional, porém apresenta uma série de limitações, não sendo utilizada em solos muito úmidos, além de não controlar as plantas daninhas na linha de plantio (Freitas et al., 2009b).

O controle químico com o uso de herbicidas apresenta diversas vantagens, como: menor dependência de mão de obra; eficiência mesmo em épocas chuvosas; eficiência no controle de plantas daninhas na linha de plantio e não afeta o sistema radicular das culturas; permite o cultivo mínimo ou plantio direto; e eficiência no controle de plantas daninhas de propagação vegetativa (Silva $\&$ Silva, 2007). No entanto, a utilização desse método na cultura do feijãocaupi é limitada devido à escassez de trabalhos envolvendo a seletividade de herbicidas nesta cultura e à falta de agrotóxicos registrados no Ministério da Agricultura, Pecuária e Abastecimento, o que impede a recomendação e o uso desses produtos (Silva \& Albertino, 2009). A maioria das informações sobre a utilização de herbicidas está relacionada à cultura do feijão-comum (Phaseolus vulgaris) e da soja (Glicyne maxima) (Freitas et al., 2009b).
Contudo, por serem espécies diferentes, pode ocorrer variação na seletividade entre as culturas mencionadas e o feijão-caupi, conforme verificado por Mesquita (2011), que constatou que as misturas metribuzin + fluazifop-p-butyl e chlorimuron-ethyl + fluazifop-p-butyl causaram a morte da cultura do feijão-caupi, apesar de seletivos para a cultura da soja.

Poucos trabalhos foram realizados avaliando a utilização de herbicidas para a cultura do feijão-caupi, em que se destacaram os seguintes herbicidas com relação à seletividade: imazamox + bentazon (Mesquita, 2011), imazamox (Silva et al., 2003; Mesquita, 2011), bentazon, fluazifop-p-butyl, imazethapyr, trifluralin, S-metolachlor (Mesquita, 2011), fenoxaprop-p-ethyl (Silva et al., 2003) e oxadiazon (Fontes et al., 2010). Severa intoxicação com redução na produtividade foi verificada no feijão-caupi quando aplicados os herbicidas fomesafen (Mesquita, 2011; Linhares et al., prelo) e lactofen (Mesquita, 2011), enquanto as misturas metribuzin e chlorimuron-ethyl + fluazifop-p-butyl causaram a morte da cultura (Mesquita, 2011).

Diante da expansão da área cultivada e da carência de mão de obra para controle de plantas daninhas, o presente trabalho objetivou avaliar a seletividade e eficácia de herbicidas para a cultura do feijão-caupi.

\section{MATERIAL E MÉTODOS}

O experimento foi conduzido no município de Mossoró-RN, no período de março a junho de 2011. O solo da área experimental é classificado como Argissolo Vermelho-Amarelo eutrófico (Santos et al., 2006), cuja análise química revelou os seguintes resultados: $\mathrm{pH}$ (água) $=7,9, \mathrm{P}=157 \mathrm{mg} \mathrm{dm}^{-3}, \mathrm{~K}=0,3 \mathrm{cmol}_{\mathrm{c}} \mathrm{dm}^{-3}$, $\mathrm{Ca}=3,2 \mathrm{cmol}_{\mathrm{c}} \mathrm{dm}^{-3}, \mathrm{Na}=0,32 \mathrm{cmol}_{\mathrm{c}} \mathrm{dm}^{-3} \mathrm{e}$ $\mathrm{Mg}=1,0 \mathrm{cmol}_{\mathrm{c}} \mathrm{dm}^{-3}$. Utilizou-se o esquema de parcelas subdivididas, distribuídas no delineamento de blocos casualizados, com quatro repetições. Nas parcelas, foram avaliadas dez estratégias de manejo de plantas daninhas, sendo nove herbicidas/misturas mais um tratamento sem herbicidas (Tabela 1), e nas subparcelas, dois tratamentos, um mantido no limpo com capinas, no intuito de avaliar a seletividade dos herbicidas à cultura em 
Tabela 1 - Relação dos herbicidas com as respectivas doses aplicadas na cultura do feijão-caupi. Mossoró, 2011

\begin{tabular}{|c|c|}
\hline Herbicida/mistura & Dose (kg ha ${ }^{-1}$ i.a. $)$ \\
\hline S-metolachlor ${ }^{1 /}$ & 1,15 \\
\hline Bentazon $^{2 /}+$ imazamox $^{2 /}$ & $1,20+0,06$ \\
\hline S-metolachlor ${ }^{1 /}+$ bentazon $^{2 /}+$ imazamox $^{2 /}$ & $1,15+1,20+0,06$ \\
\hline Imazamox $^{\underline{2} /}+$ fluazifop-p-butyl ${ }^{3 /}$ & $0,04+0,12$ \\
\hline Imazethapyr $^{\underline{2} /}+$ fluazifop-p-butyl $^{3 /}$ & $0,10+0,12$ \\
\hline 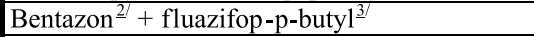 & $1,20+0,12$ \\
\hline Bentazon $^{2 /}+$ imazamox $^{2 /}+$ fluazifop-p-butyl $^{3 /}$ & $1,20+0,06+0,12$ \\
\hline Lactofen $^{2 /}+$ fluazifop-p-butyl ${ }^{3 /}$ & $0,17+0,12$ \\
\hline Fluazifop -p-butyl ${ }^{\underline{3} /}$ & 0,12 \\
\hline Testemunha sem herbicida $\mathrm{s}$ & ---- \\
\hline
\end{tabular}

ausência das plantas daninhas, e outro sem capinas, visando avaliar a eficácia dos herbicidas no controle das plantas infestantes.

Cada parcela experimental se constituiu de quatro fileiras de $5 \mathrm{~m}$ de comprimento, espaçadas de 0,6 m entre si. A área útil de cada parcela foi composta pelas duas fileiras centrais, descartando-se 0,5 m em cada uma das extremidades.

O preparo do solo foi realizado no sistema convencional, com uma aração e duas gradagens. A adubação de plantio foi feita com base na análise química do solo, utilizando-se $200 \mathrm{~kg} \mathrm{ha}^{-1}$ da formulação N-P-K 06-24-12. A semeadura foi realizada manualmente, com dez sementes por metro linear de fileira, no dia 4 de março de 2011. O cultivar utilizado foi o BRS Guariba, de porte semiereto, grãos brancos, com ciclo em torno de dias (Vilarinho, 2007).

O herbicida S-metolachlor foi aplicado em pré-emergência da cultura e das plantas daninhas um dia após o plantio do feijão-caupi, ao passo que a aplicação dos herbicidas bentazon, imazamox, bentazon + imazamox, imazethapyr e lactofen foi feita em pós-emergência aos 14 dias após o plantio (DAP), quando a cultura estava com a segunda folha trifoliolada completamente expandida. O herbicida fluazifopp-butyl foi aplicado aos 19 DAP, devido à incompatibilidade fisiológica com outros herbicidas (Silva \& Silva, 2007). A aplicação foi realizada utilizando-se um pulverizador costal equipado com barra com dois bicos XR 11002, espaçados de $50 \mathrm{~cm}$, mantidos a uma altura de $50 \mathrm{~cm}$ do alvo, sob pressão de $250 \mathrm{kPa}$, com volume de calda de $200 \mathrm{~L} \mathrm{ha}^{-1}$. Durante a aplicação, a fim de evitar deriva, as parcelas vizinhas foram protegidas lateralmente com uma placa de madeira.

A seletividade dos herbicidas para a cultura foi determinada por meio de avaliações visuais de intoxicação aos 21,28 e 45 DAP, utilizando-se a escala de 1 a 4, em que 1 representa ausência de intoxicação; 2, intoxicação leve; 3 , intoxicação moderada; e 4, intoxicação severa.

A eficácia dos herbicidas no controle de plantas daninhas foi determinada, para cada espécie infestante, por meio de avaliações visuais aos 21 e 28 DAP, atribuindo-se notas de 0 a 100 , em que 0 indica ausência de controle e 100, controle total das plantas daninhas em relação ao tratamento sem herbicidas e sem capinas, conforme procedimentos da Sociedade Brasileira da Ciência das Plantas Daninhas (SBCPD, 1995).

Aos 45 DAP, foram feitas avaliações de massa seca de plantas daninhas nas subparcelas sem capinas, por meio de uma amostragem em quadrado com $0,5 \mathrm{~m}$ de lado $\left(0,25 \mathrm{~m}^{2}\right)$ por subparcela. As plantas daninhas foram colhidas ao nivel do solo, separadas por espécie, contadas e levadas à estufa com circulação forçada de ar à temperatura de $65^{\circ} \mathrm{C}$, até obter massa constante, para determinação da massa matéria seca.

Avaliou-se, para o feijão-caupi, o número de dias para florescimento e colheita em relação à emergência; a determinação da data de florescimento foi realizada quando $50 \%$ das plantas estavam floridas. Já a determinação da data de colheita foi feita quando as plantas estavam com $80 \%$ das vagens secas. Por ocasião da colheita, foram avaliados o número de vagens por planta, o peso de 100 grãos e a produtividade de grãos, com umidade corrigida para $13 \%$, expressa em $\mathrm{kg} \mathrm{ha}^{-1}$, obtida a partir da colheita das plantas da área útil das subparcelas.

Os dados climatológicos (temperaturas máxima, média e mínima e precipitação) durante o período experimental estão apresentados na Figura 1.

Os dados obtidos foram submetidos à análise de variância pelo teste $\mathrm{F}$ e, em caso 

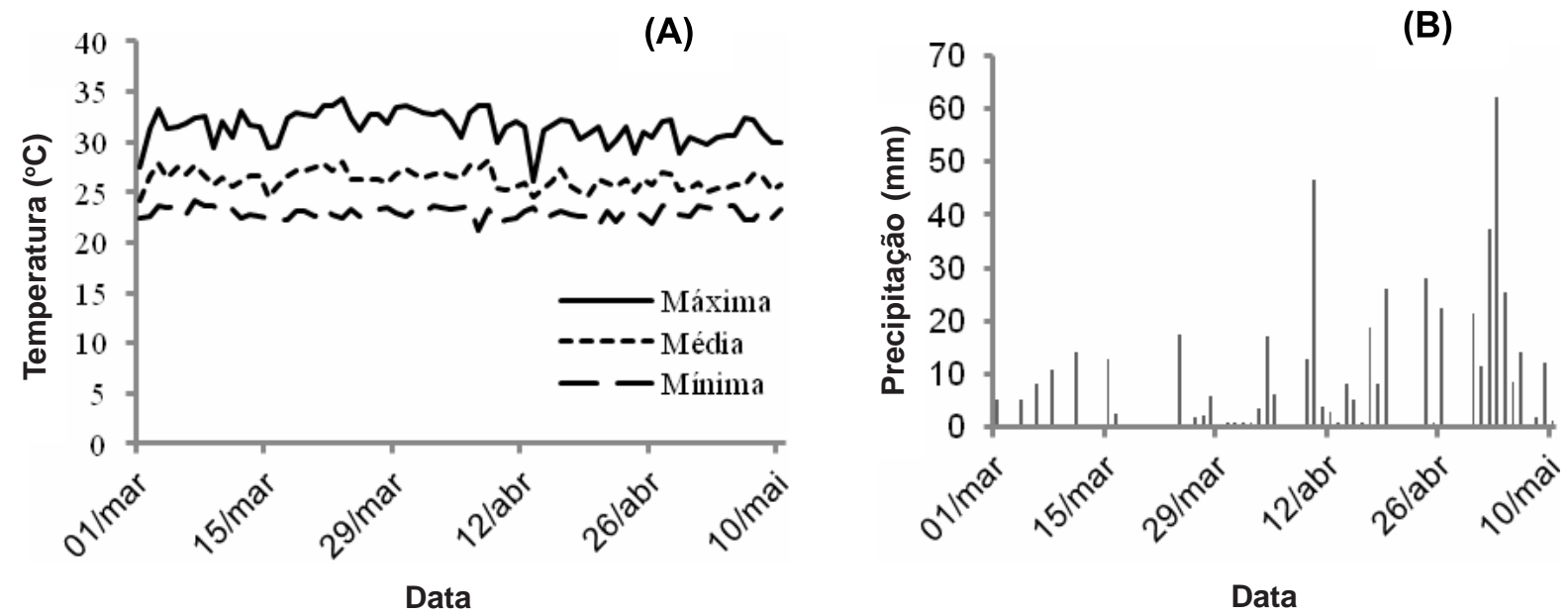

Figura 1 - Temperaturas máxima, média e mínima do ar (A) e precipitação (B) durante o ciclo da cultura. Estação meteorológica da UFERSA. Mossoró-RN, 2011.

de significância, foram comparados através do teste de Duncan a $5 \%$ de probabilidade. Os dados referentes à massa seca de plantas daninhas foram transformados em $\sqrt{y+1}$, de acordo com Ferreira (1991), visando atender às pressuposições da análise de variância.

\section{RESULTADOS E DISCUSSÃO}

Houve variação no nível de intoxicação entre os herbicidas avaliados sobre a cultura do feijão-caupi (Tabela 2), tendo-se constatado aos 21 DAP apenas sintomas leves para os herbicidas bentazon + imazamox, $\mathrm{S}$-metolachlor + bentazon + imazamox, imazamox + fluazifop-p-butyl, imazethapyr + fluazifop-p-butyl, bentazon + fluazifop-p-butyl e bentazon + imazamox + fluazifop-p-butyl, com recuperação total da planta aos 28 DAP. Resultados semelhantes foram verificados por Freitas et al. (2010) para os herbicidas S-metolachlor, fluazifop-p-butyl, imazethapyr, imazamox, bentazon e imazamox + bentazon e por Mesquita (2011), trabalhando com bentazon + fluazifop-p-butyl, imazamox + fluazifop-pbutyl, imazamox + bentazon + fluazifop-p-butyl e imazethapyr + fluazifop-p-butyl.

Verificou-se também (Tabela 2) que maiores niveis de intoxicação foram observados aos 21 DAP, quando se fez a aplicação do tratamento lactofen + fluazifop-p-butyl, que corresponde a sete e dois dias após a aplicação dos herbicidas lactofen e fluazifop-p-butyl, respectivamente. Os sintomas foram caracterizados por necrose no limbo foliar. No entanto, apesar da severa intoxicação, a gema apical não foi afetada e os sintomas de intoxicação desapareceram com o desenvolvimento das plantas, devido à emissão e expansão de folhas novas, com total recuperação aos 45 DAP.

Correa \& Alves (2009) verificaram que, para a cultura da soja, o herbicida lactofen em mistura com outros herbicidas causou apenas intoxicação leve; os sintomas tornaram-se menos intensos após os 13 DAA. Também para a cultura da soja, Souza et al. (2002), trabalhando com diferentes doses do herbicida lactofen, observaram que a fitointoxicação provocada pelo lactofen isolado atingiu valores intermediários no terceiro dia após a aplicação; contudo, com o desenvolvimento da cultura, os sintomas se tornaram menos intensos até o décimo dia após a aplicação e, a partir do vigésimo dia, desapareceram completamente.

No tratamento com aplicação do fluazifopp-butyl, que é um herbicida inibidor da enzima Acetil Coenzima-A Carboxilase (ACCase), específico para o controle de plantas daninhas gramíneas (Silva \& Silva, 2007), não se verificaram sintomas de intoxicação ou alteração no ciclo do feijão-caupi (Tabelas 2 e 3), o que demonstra a seletividade desse herbicida para a cultura, conforme constatado por Freitas et al. (2010) e Mesquita (2011). Assim, 
Tabela 2 - Intoxicação de plantas de feijão-caupi aos 21, 28 e 45 dias após o plantio (DAP). Mossoró, 2011

\begin{tabular}{|c|c|c|c|}
\hline Herbicida/mistura & 21 DAP & 28 DAP & 45 DAP \\
\hline S-metolachlor ${ }^{1 /}$ & $1,0 \mathrm{~d}^{*}$ & $1,0 \mathrm{~b}$ & $1,0 \mathrm{a}$ \\
\hline Bentazon $^{2 /}+$ imazamox $^{2 /}$ & $2,0 \mathrm{~b}$ & $1,0 \mathrm{~b}$ & $1,0 \mathrm{a}$ \\
\hline S-metolachlor ${ }^{1 /}+$ bentazon $^{2 /}+$ imazamox $^{\underline{2} /}$ & $1,9 \mathrm{~b}$ & $1,3 \mathrm{~b}$ & $1,0 \mathrm{a}$ \\
\hline Imazamox $^{2 /}+$ fluazifop-p-butyl ${ }^{3 /}$ & $1,9 \mathrm{~b}$ & $1,0 \mathrm{~b}$ & $1,0 \mathrm{a}$ \\
\hline Imazethapyr $^{2 /}+$ fluazifop-p-butyl $^{3 / /}$ & $1,5 \mathrm{c}$ & $1,0 \mathrm{~b}$ & $1,0 \mathrm{a}$ \\
\hline Bentazon $^{2 /}+$ fluazifop-p-butyl $l^{3 /}$ & $1,9 \mathrm{~b}$ & $1,0 \mathrm{~b}$ & $1,0 \mathrm{a}$ \\
\hline Bentazon $^{2 /}+$ imazamox $^{2 /}+$ fluazifop-p-butyl $^{3 /}$ & $2,0 \mathrm{~b}$ & $1,0 \mathrm{~b}$ & $1,0 \mathrm{a}$ \\
\hline Lactofen $^{2 /}+$ fluazifop-p-butyl $1^{3 /}$ & $3,3 \mathrm{a}$ & $2,6 \mathrm{a}$ & $1,0 \mathrm{a}$ \\
\hline Fluazifop-p-butyl $\mathbf{3}^{3 /}$ & $1,0 \mathrm{~d}$ & $1,0 \mathrm{~b}$ & $1,0 \mathrm{a}$ \\
\hline Testemunha sem herbicidas & $1,0 \mathrm{~d}$ & $1,0 \mathrm{~b}$ & $1,0 \mathrm{a}$ \\
\hline
\end{tabular}

* Nas colunas, médias seguidas de mesmas letras não diferem estatisticamente pelo teste de Duncan a 5\% de probabilidade. 1- ausência de intoxicação; 2- intoxicação leve; 3- intoxicação moderada; e 4- intoxicação severa. ${ }^{1 /}$ Aplicação um dia após o plantio (DAP); $\stackrel{2}{ }$ aplicação

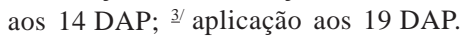

Tabela 3 - Época de floração e colheita do feijão-caupi em dias após a emergência (DAE), em função da aplicação de herbicidas. Mossoró, 2011

\begin{tabular}{|c|c|c|}
\hline Herbicida/mistura & Floração (DAE) & Colheita (DAE) \\
\hline S-metolachlor ${ }^{1 /}$ & $42 \mathrm{a}^{*}$ & $62 \mathrm{a}$ \\
\hline Bentazon $^{2 /}+$ imazamox $^{2 /}$ & $43 \mathrm{a}$ & $66 \mathrm{a}$ \\
\hline S-metolachlor ${ }^{1 /}+$ bentazon $^{2 /}+$ imazamox $^{2 /}$ & $43 \mathrm{a}$ & $65 \mathrm{a}$ \\
\hline Imazamox $^{2 /}+$ fluazifop-p-butyl ${ }^{3 /}$ & $43 \mathrm{a}$ & $65 \mathrm{a}$ \\
\hline Imazethapyr $^{2 /}+$ fluazifop-p-butyl $1^{3 /}$ & $43 \mathrm{a}$ & $64 \mathrm{a}$ \\
\hline Bentazon $^{2 /}+$ fluazifop-p-butyl $l^{3 /}$ & $42 \mathrm{a}$ & $63 \mathrm{a}$ \\
\hline Bentazon $^{2 /}+$ imazamox $^{2 /}+$ fluazifop-p-butyl $l^{3 /}$ & $43 \mathrm{a}$ & $64 \mathrm{a}$ \\
\hline Lactofen $^{2 /}+$ fluazifop-p-butyl ${ }^{3 /}$ & $50 \mathrm{~b}$ & $70 \mathrm{~b}$ \\
\hline Fluazifop-p-butyl ${ }^{3 /}$ & $42 \mathrm{a}$ & $63 \mathrm{a}$ \\
\hline Testemunha sem herbicidas & $42 \mathrm{a}$ & $62 \mathrm{a}$ \\
\hline
\end{tabular}

* Nas colunas, médias seguidas de mesma letra não diferem entre si pelo teste de Duncan a $5 \%$ de probabilidade. ${ }^{1 / 1}$ Aplicação um dia após o plantio (DAP); ำ aplicação aos 14 DAP; ’ aplicação aos 19 DAP.

pode-se inferir que a intoxicação e alteração no ciclo do feijão-caupi foram causadas pelo lactofen. Freitas et al. (2010), avaliando a aplicação do lactofen em feijão-caupi, constataram niveis de intoxicação semelhantes aos observados neste trabalho com a aplicação em mistura com fluazifop-p-butyl.

Os danos causados pela intoxicação da mistura lactofen + fluazifop-p-butyl se refletiram no desenvolvimento da cultura, resultando em prolongamento no ciclo; a floração e colheita ocorreram oito dias após a testemunha sem herbicidas (Tabela 3), corroborando os resultados mostrados por Mesquita (2011), que verificou que a aplicação da mistura lactofen + fluazifop-p-butyl retardou a floração e a colheita em nove dias após a testemunha sem herbicidas.
As principais espécies de plantas daninhas que ocorreram na área foram Cleome affinis, Trianthema portulacastrum, Amaranthus spinosus, Digitaria bicornis e Commelina benghalensis, sendo todas elas controladas com eficácia pelos herbicidas S-metolachlor + bentazon + imazamox e lactofen + fluazifop-pbutyl aos 28 DAP (Tabela 4). O S-metolachlor controlou de modo eficiente todas as espécies presentes, incluindo gramíneas e dicotiledôneas, com exceção de $C$. affinis, o que está de acordo com Silva \& Silva (2007), os quais relatam que este herbicida controla com eficiência plantas daninhas da família Poaceae e algumas dicotiledôneas das famílias Amaranthaceae e Commelinaceae, como é o caso de A. spinosus e C. benghalensis, respectivamente. Mesquita (2011) também constatou eficácia do S-metolachlor para as 
respectivas espécies e nenhuma eficiência para C. affinis.

O fluazifop-p-butyl, aplicado isoladamente, exerceu efeito apenas sobre $D$. bicornis, como pode ser observado na Tabela 4; aos 21 DAP, que corresponde a dois dias após a aplicação desse herbicida, o efeito sobre essa espécie foi moderado, atingindo controle total sete dias depois (28 DAP), concordando com Rodrigues \& Almeida (2011), segundo os quais herbicidas inibidores de ACCase exercem efeito apenas sobre plantas daninhas gramíneas.

O imazamox + fluazifop-p-butyl e o bentazon + fluazifop-p-butyl não controlaram satisfatoriamente $T$. portulacastrum e A. spinosus, respectivamente; já a mistura bentazon + imazamox + fluazifop-p-butyl potencializou o controle de ambas as espécies, proporcionando controle de $T$. portulacastrum na ordem de $60 \%$ e total controle de $A$. spinosus aos 28 DAP (Tabela 4). Freitas et al. (2009b) e Mesquita (2011) também verificaram que o bentazon isoladamente ou em mistura com fluazifop-p-butyl, respectivamente, mostrou-se ineficiente no controle de A. spinosus.

Com relação à massa seca de plantas daninhas (Tabela 5), observou-se, aos 45 DAP, que os tratamentos S-metolachlor, bentazon + fluazifop-p-butyl e fluazifop-p-butyl proporcionaram maior acúmulo de massa seca total das plantas infestantes, assim como a testemunha sem capinas, isso porque os herbicidas S-metolachlor e bentazon + fluazifop-p-butyl não exerceram controle eficiente sobre C. affinis e A. spinosus, respectivamente (Tabela 4), e o fluazifop-p-butyl não exerce efeito sobre as plantas dicotiledôneas.

A aplicação dos demais herbicidas resultou na redução da massa seca de plantas daninhas em comparação com a testemunha sem capinas; o menor acúmulo foi verificado nos tratamentos com S-metolachlor + bentazon + imazamox e lactofen + fluazifop-p-butyl

Tabela 4 - Porcentagem de controle de plantas daninhas na cultura do feijão-caupi submetida à aplicação de diferentes herbicidas, aos 21 e 28 dias após o plantio. Mossoró, 2011’

\begin{tabular}{|c|c|c|c|c|c|}
\hline Herbicida/mistura & C. affinis & $\begin{array}{c}T . \\
\text { portulacastrum }\end{array}$ & A. spinosus & D. bicornis & $\begin{array}{c}C . \\
\text { benghalensis }\end{array}$ \\
\hline \multicolumn{6}{|c|}{21 dias após o plantio } \\
\hline S-metolachlor ${ }^{1 /}$ & $0,00 b^{*}$ & $100,00 \mathrm{a}$ & $100,00 \mathrm{a}$ & $100,00 \mathrm{a}$ & $100,00 \mathrm{a}$ \\
\hline Bentazon $^{2 /}+$ imazamox $^{\underline{2} /}$ & $100,00 \mathrm{a}$ & $37,50 \mathrm{~b}$ & $100,00 \mathrm{a}$ & $8,75 \mathrm{~d}$ & $100,00 \mathrm{a}$ \\
\hline S-metolachlor ${ }^{1 /}+$ bentazon $^{2 /}+$ imazamox $^{2 /}$ & $100,00 \mathrm{a}$ & $100,00 \mathrm{a}$ & $100,00 \mathrm{a}$ & $100,00 \mathrm{a}$ & $75,00 \mathrm{a}$ \\
\hline Imazamox $^{2 /}+$ fluazifop-p-butyl ${ }^{3 /}$ & $83,75 \mathrm{a}$ & $25,00 \mathrm{bc}$ & $100,00 \mathrm{a}$ & $83,75 \mathrm{ab}$ & $100,00 \mathrm{a}$ \\
\hline Imazethapyr $^{2 /}+$ fluazifop-p-butyl ${ }^{3 /}$ & $88,75 \mathrm{a}$ & $31,25 \mathrm{~b}$ & $100,00 \mathrm{a}$ & $100,00 \mathrm{a}$ & $100,00 \mathrm{a}$ \\
\hline Bentazon $^{2 /}+$ fluazifop-p-butyl $l^{3 /}$ & $95,00 \mathrm{a}$ & $32,50 \mathrm{~b}$ & $15,00 \mathrm{~b}$ & $72,50 \mathrm{~b}$ & $100,00 \mathrm{a}$ \\
\hline Bentazon $^{2 /}+$ imazamox $^{\underline{2} /}+$ fluazifop-p-butyl $l^{3 /}$ & $100,00 \mathrm{a}$ & $32,50 \mathrm{~b}$ & $100,00 \mathrm{a}$ & $71,25 \mathrm{~b}$ & $100,00 \mathrm{a}$ \\
\hline Lactofen $^{2 /}+$ fluazifop-p-butyl $l^{3 /}$ & $99,75 \mathrm{a}$ & $98,75 \mathrm{a}$ & $99,50 \mathrm{a}$ & $62,50 \mathrm{bc}$ & $99,00 \mathrm{a}$ \\
\hline Fluazifop-p-butyl ${ }^{3 /}$ & $0,00 \mathrm{~b}$ & $0,00 \mathrm{c}$ & $0,00 \mathrm{c}$ & $43,75 \mathrm{c}$ & $0,00 \mathrm{~b}$ \\
\hline \multicolumn{6}{|c|}{28 dias após o plantio } \\
\hline S-metolachlor $^{1 /}$ & $0,00 \mathrm{~b}$ & $100,00 \mathrm{a}$ & $100,00 \mathrm{a}$ & $100,00 \mathrm{a}$ & $100,00 \mathrm{a}$ \\
\hline Bentazon $^{2 /}+$ imazamox $^{2 /}$ & $100,00 \mathrm{a}$ & $65,00 \mathrm{abc}$ & $100,00 \mathrm{a}$ & $0,00 \mathrm{~b}$ & $100,00 \mathrm{a}$ \\
\hline S-metolachlor $^{1 /}+$ bentazon $^{2 /}+$ imazamox $^{2 /}$ & $100,00 \mathrm{a}$ & $100,00 \mathrm{a}$ & $100,00 \mathrm{a}$ & $100,00 \mathrm{a}$ & $100,00 \mathrm{a}$ \\
\hline Imazamox $^{2 /}+$ fluazifop-p-butyl $l^{3 /}$ & $100,00 \mathrm{a}$ & $33,75 \mathrm{~cd}$ & $100,00 \mathrm{a}$ & $100,00 \mathrm{a}$ & $92,50 \mathrm{a}$ \\
\hline Imazethapyr $^{2 /}+$ fluazifop-p-butyl ${ }^{3 /}$ & $100,00 \mathrm{a}$ & 70,00 abc & $100,00 \mathrm{a}$ & $99,50 \mathrm{a}$ & $100,00 \mathrm{a}$ \\
\hline Bentazon $^{2 /}+$ fluazifop-p-butyl ${ }^{3 /}$ & $100,00 \mathrm{a}$ & $56,25 \mathrm{~cd}$ & $13,75 \mathrm{~b}$ & $100,00 \mathrm{a}$ & $100,00 \mathrm{a}$ \\
\hline Bentazon $^{2 /}+$ imazamox $^{2 /}+$ fluazifop-p-butyl $^{3 /}$ & $100,00 \mathrm{a}$ & $63,00 \mathrm{bc}$ & $100,00 \mathrm{a}$ & $100,00 \mathrm{a}$ & $100,00 \mathrm{a}$ \\
\hline Lactofen $^{2 /}+$ fluazifop-p-butyl ${ }^{3 /}$ & $100,00 \mathrm{a}$ & $97,00 \mathrm{ab}$ & $100,00 \mathrm{a}$ & $100,00 \mathrm{a}$ & $89,50 \mathrm{a}$ \\
\hline${\text { Fluazifop-p-butyl }{ }^{3 /}}^{3}$ & $0,00 \mathrm{~b}$ & $0,00 \mathrm{~d}$ & $0,00 \mathrm{c}$ & $100,00 \mathrm{a}$ & $0,00 \mathrm{~b}$ \\
\hline
\end{tabular}

* Nas colunas, médias seguidas de mesma letra não diferem entre si pelo teste de Duncan a 5\% de probabilidade, ${ }^{1 /}$ Aplicação um dia após o plantio (DAP); ’a aplicação aos 14 DAP; ’ aplicação aos 19 DAP. 
Tabela 5 - Massa seca de plantas daninhas $\left(\mathrm{g} \mathrm{m}^{-2}\right.$ ) na cultura do feijão-caupi aos 45 dias após o plantio (DAP). Mossoró, 2011

\begin{tabular}{|c|c|c|c|c|c|c|}
\hline Herbicida/mistura & C. affinis & $\begin{array}{c}T . \\
\text { portulacastrum }\end{array}$ & A. spinosus & D. bicornis & $\begin{array}{c}C . \\
\text { benghalensis }\end{array}$ & Total \\
\hline S-metolachlor ${ }^{1 /}$ & $4,19(16,54) \mathrm{a}^{*}$ & $2,21(3,90) \mathrm{ab}$ & $1,01(0,03) \mathrm{b}$ & $1,00(0,00) \mathrm{b}$ & $1,00(0,00) \mathrm{b}$ & $5,41(28,25) \mathrm{ab}$ \\
\hline Bentazon $^{2 /}+$ imazamox $^{2 /}$ & $1,00(0,00) \mathrm{b}$ & $3,38(10,45) \mathrm{a}$ & $1,00(0,00) \mathrm{b}$ & $1,05(0,10) \mathrm{a}$ & $1,00(0,00) \mathrm{b}$ & $3,40(10,62) \mathrm{c}$ \\
\hline S-metolachlor ${ }^{1 /}+$ bentazon $^{2 /}+$ imazamox $^{2 /}$ & $1,00(0,00) \mathrm{b}$ & $1,00(0,00) \mathrm{b}$ & $1,00(0,00) \mathrm{b}$ & $1,00(0,00) \mathrm{b}$ & $1,00(0,00) \mathrm{b}$ & $1,00(0,00) \mathrm{d}$ \\
\hline Imazamox $^{2 /}+$ fluazifop-p-buty $]^{3 /}$ & $1,10(0,22) \mathrm{b}$ & $3,88(14,09) \mathrm{a}$ & $1,00(0,00) \mathrm{b}$ & $1,00(0,00) \mathrm{b}$ & $1,03(0,07) \mathrm{b}$ & $3,95(14,60) \mathrm{c}$ \\
\hline Imazethapyr $^{2^{\prime}}+$ fluazifop-p-butyl $1^{3 /}$ & $1,92(2,70) \mathrm{b}$ & $3,95(14,55) \mathrm{a}$ & $1,00(0,00) \mathrm{b}$ & $1,00(0,00) \mathrm{b}$ & $1,00(0,00) \mathrm{b}$ & $4,52(19,45) \mathrm{b}$ \\
\hline Bentazon $^{2 /}+$ fluazifop-p-butyl $l^{3 /}$ & $1,65(1,72) \mathrm{b}$ & $3,40(10,60) \mathrm{a}$ & $2,70(6,30) \mathrm{a}$ & $1,00(0,00) b$ & $1,00(0,00) \mathrm{b}$ & $4,69(21,00) \mathrm{b}$ \\
\hline Bentazon $^{2 /}+$ imazamox $^{2 /}+$ fluazifop-p-butyl ${ }^{3 /}$ & $1,26(0,59) \mathrm{b}$ & $3,97(14,78) \mathrm{a}$ & $1,35(0,83) \mathrm{b}$ & $1,00(0,00) \mathrm{b}$ & $1,00(0,00) \mathrm{b}$ & $4,21(16,75) \mathrm{bc}$ \\
\hline Lactofen $^{2 /}+$ fluazifop -p-butyl $l^{3 /}$ & $1,00(0,00) \mathrm{b}$ & $1,00(0,00) \mathrm{b}$ & $1,00(0,00) \mathrm{b}$ & $1,00(0,00) \mathrm{b}$ & $1,00(0,00) \mathrm{b}$ & $1,00(0,00) \mathrm{d}$ \\
\hline Fluazifop-p-butyl ${ }^{3 / 2}$ & $4,03(15,28) \mathrm{a}$ & $3,59(11,89) \mathrm{a}$ & $1,55(1,40) \mathrm{b}$ & $1,00(0,00) \mathrm{b}$ & $1,09(0,20) \mathrm{ab}$ & $6,08(35,98) \mathrm{a}$ \\
\hline Testemunha sem herbicidas & $4,13(15,10) \mathrm{a}$ & $2,67(6,15) \mathrm{ab}$ & $1,08(0,17) \mathrm{b}$ & $1,04(0,09) \mathrm{a}$ & $1,23(0,51) \mathrm{a}$ & $5,08(24,86) \mathrm{ab}$ \\
\hline
\end{tabular}

* Nas colunas, médias seguidas de mesma letra não diferem entre si pelo teste de Duncan a 5\% de probabilidade; os dados foram

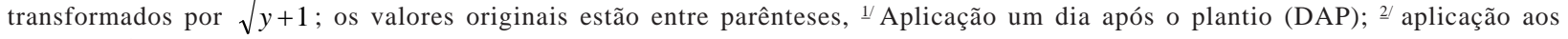
14 DAP; ’ aplicação aos 19 DAP.

(Tabela 5), visto que esses dois tratamentos apresentaram controle eficiente sobre todas as espécies presentes (Tabela 4). No entanto, os tratamentos imazethapyr + fluazifop-p-butyl e bentazon + imazamox + fluazifop-p-butyl, que resultaram em acúmulo de massa seca intermediário, poderiam ser mais eficientes em áreas sem ocorrência da espécie T. portulacastrum, a qual não foi controlada com eficácia pelas respectivas misturas, uma vez que a eficácia e, consequentemente, a escolha dos herbicidas dependem das espécies infestantes, que, segundo Marques et al. (2011), variam no decorrer dos ciclos produtivos em razão das alterações das culturas e das práticas de manejo.

Verificou-se interação significativa entre as estratégias de manejo de plantas daninhas envolvendo o uso de herbicidas e sistemas com ou sem capinas para as variáveis número de vagens por planta e produtividade, as quais se comportaram de modo semelhante quando os tratamentos com herbicidas foram submetidos a capina. Não se observou diferença entre os herbicidas, que se comportaram de forma semelhante à da testemunha, com exceção do tratamento com aplicação do lactofen + fluazifop-p-butyl, que apresentou maior número de vagens por planta e produtividade em relação aos demais (Tabela 6). Apesar da severa intoxicação (Tabela 2) e atraso no ciclo da cultura (Tabela 3), a aplicação desta mistura pode ter influenciado positivamente os componentes da produtividade devido às condições ambientais, com temperaturas elevadas e alto índice pluviométrico, verificadas na segunda metade do ciclo (Figura 1A, B), proporcionando intenso crescimento vegetativo na cultura. Em condições ambientais adequadas para a cultura, não se espera que a intoxicação causada por essa mistura seja benéfica, conforme resultados obtidos por Mesquita (2011), o qual verificou que a intoxicação causada pela mistura lactofen + fluazifop-p-buty resultou em queda na produtividade.

Quando se avaliaram os tratamentos com herbicidas sem a realização de capinas (Tabela 6), constatou-se maior número de vagens por planta e produtividade quando se procedeu à aplicação do lactofen + fluazifop-pbutyl, em relação aos demais tratamentos, devido ao excelente nível de controle das plantas daninhas e também ao efeito do lactofen, atuando como regulador de crescimento, ao passo que os tratamentos compostos pelo bentazon + fluazifop-p-butyl, fluazifop-p-butyl e pela testemunha sem herbicidas apresentaram produtividade inferior à das demais estratégias de manejo e seus respectivos tratamentos com capinas, devido à interferência exercida pelas plantas daninhas não controladas. Mesquita (2011) também constatou que os herbicidas imazethapyr + fluazifop-p-butyl e imazamox + bentazon + fluazifop-p-butyl proporcionaram número de vagens por planta e produtividade equivalentes aos das testemunha capinada. 
Tabela 6 - Número de vagens por planta, peso de cem grãos e produtividade do feijão-caupi em função das estratégias de manejo de plantas daninhas. Mossoró, 2011

\begin{tabular}{|c|c|c|c|c|c|c|}
\hline \multirow[t]{2}{*}{ Herbicida/mistura } & \multicolumn{2}{|c|}{$\begin{array}{l}\text { Número de vagens } \\
\text { por planta }\end{array}$} & \multicolumn{2}{|c|}{ Peso de cem grãos (g) } & \multicolumn{2}{|c|}{ Produtividade $\left(\mathrm{kg} \mathrm{ha}^{-1}\right)$} \\
\hline & Com capinas & Sem capinas & Com capinas & Sem capinas & Com capinas & Sem capinas \\
\hline S-metolachlor $^{1 /}$ & $5,0 \mathrm{bA} *$ & $4,8 \mathrm{abA}$ & $15,79 \mathrm{aA}$ & $17,03 \mathrm{aA}$ & $825,7 \mathrm{bA}$ & $808,6 \mathrm{abA}$ \\
\hline Bentazon $^{2 /}+$ imazamox $^{2 /}$ & $5,3 \mathrm{bA}$ & $4,7 \mathrm{abA}$ & $17,45 \mathrm{aA}$ & $18,53 \mathrm{aA}$ & $906,5 \mathrm{bA}$ & $791,6 \mathrm{abA}$ \\
\hline S-metolachlor ${ }^{1 /}+$ bentazon $^{2 /}+$ imazamox $^{2 /}$ & $5,2 \mathrm{bA}$ & $6,0 \mathrm{aA}$ & $18,36 \mathrm{aA}$ & $17,51 \mathrm{aA}$ & $876,7 \mathrm{bA}$ & $1017,2 \mathrm{abA}$ \\
\hline Imazamox $^{2 /}+$ fluazifop-p-butyl ${ }^{3 /}$ & $5,2 \mathrm{bA}$ & $5,4 \mathrm{abA}$ & $18,73 \mathrm{aA}$ & $17,07 \mathrm{aA}$ & $885,2 \mathrm{bA}$ & $923,6 \mathrm{abA}$ \\
\hline Imazethapyr $^{2 /}+$ fluazifop-p-butyl $1^{3 /}$ & $5,4 \mathrm{bA}$ & $5,4 \mathrm{abA}$ & $16,60 \mathrm{aA}$ & $16,22 \mathrm{aA}$ & $923,6 \mathrm{bA}$ & $919,3 \mathrm{abA}$ \\
\hline Bentazon $^{2 /}+$ fluazifop-p-butyl ${ }^{3 /}$ & $5,5 \mathrm{bA}$ & $3,7 \mathrm{bB}$ & $17,90 \mathrm{aA}$ & $17,90 \mathrm{aA}$ & $940,6 \mathrm{bA}$ & $621,4 \mathrm{bB}$ \\
\hline Bentazon $^{2 /}+$ imazamox $^{2 /}+$ fluazifop-p-butyl $l^{3 /}$ & $4,8 \mathrm{bA}$ & $5,1 \mathrm{abA}$ & $16,37 \mathrm{aA}$ & $17,69 \mathrm{aA}$ & $817,2 \mathrm{bA}$ & 864,0 abA \\
\hline Lactofen $^{2 /}+$ fluazifop-p-butyl ${ }^{3 /}$ & $7,1 \mathrm{aA}$ & $6,6 \mathrm{aA}$ & $19,82 \mathrm{aA}$ & $19,16 \mathrm{aA}$ & $1208,7 \mathrm{aA}$ & $1123,6 \mathrm{aA}$ \\
\hline Fluazifop-p-butyl $\mathrm{l}^{3 /}$ & $4,9 \mathrm{bA}$ & $3,6 \mathrm{bB}$ & $16,28 \mathrm{aA}$ & $17,96 \mathrm{aA}$ & $834,2 \mathrm{bA}$ & $617,1 \mathrm{bB}$ \\
\hline Testemunha sem herbicidas & $5,3 \mathrm{bA}$ & $3,3 \mathrm{bB}$ & $17,59 \mathrm{aA}$ & $17,43 \mathrm{aA}$ & $902,3 \mathrm{bA}$ & $621,4 \mathrm{bB}$ \\
\hline
\end{tabular}

* Nas colunas, letras minúsculas comparam as estratégias de manejo de plantas daninhas dentro de cada sistema (com ou sem capinas) pelo teste de Duncan a 5\% de probabilidade; nas linhas, para cada característica avaliada, letras maiúsculas comparam os sistemas (com ou sem capinas dentro de estratégia de manejo de plantas daninhas) pelo teste $\mathrm{F}$ a $5 \%$ de probabilidade, ${ }^{1 /}$ Aplicação um dia após o plantio (DAP);

느 aplicação aos 14 DAP; $\underline{3}$ aplicação aos 19 DAP.

No entanto, a variável peso de cem grãos não foi afetada pelos herbicidas aplicados, independentemente do grau de intoxicação causada pelos herbicidas ou da interferência exercida pelas plantas daninhas nos tratamentos sem capinas (Tabela 6). Alguns trabalhos, avaliando interferência de plantas daninhas (Freitas et al., 2009a) e seletividade de herbicidas sobre a cultura do feijão-caupi (Mesquita, 2011; Linhares et al. Prelo), constataram que, apesar da interferência das plantas infestantes e da intoxicação dos herbicidas sobre a redução no número de vagens por planta e na produtividade, o peso de cem grãos não foi influenciado, demonstrando que esta característica é inerente ao cultivar, não sendo influenciada pelas condições de manejo, e que a queda no rendimento de grãos está relacionada, segundo Freitas et al. (2009a), a outras variáveis, como número vagens por planta e número de grãos por vagem.

Este trabalho foi conduzido no sistema de plantio convencional, com revolvimento do solo, porém os resultados observados com relação à seletividade e eficácia no controle das plantas daninhas para os herbicidas aplicados em pós-emergência podem ser extrapolados para o sistema conservacionista (plantio direto), empregado nessa cultura na região Centro-Oeste do Brasil.

Diante do exposto, conclui-se que os herbicidas S-metolachlor, bentazon + imazamox,
S-metolachlor + bentazon + imazamox, imazamox + fluazifop-p-butyl, imazethapyr + fluazifop-p-butyl, bentazon + fluazifop-p-butyl, bentazon + imazamox + fluazifop-p-butyl e fluazifop-p-butyl são seletivos para a cultura do feijão-caupi, ao passo que a aplicação dos herbicidas lactofen + fluazifop-p-butyl causou severa intoxicação na cultura, que se recuperou posteriormente, apresentando produtividade igual ou superior à dos demais tratamentos. A eficácia de herbicidas ou misturas de herbicidas para o controle de plantas daninhas pode variar de acordo com a comunidade infestante.

\section{LITERATURA CITADA}

CORRÊA, M. J. P.; ALVES, P. L. C. A. Eficácia de herbicidas aplicados em pós-emergência na cultura da soja convencional e transgênica. Planta Daninha, v. 27, p. 1035-1046, 2009.

(Número Especial)

FERREIRA, P. V. Estatística experimental aplicada à agronomia. Maceió: EDUFAL, 1991. 437 p.

FREITAS, F. C. L. et al. Interferência de plantas daninhas na cultura do feijão-caupi. Planta Daninha, v. 27, n. 2, p. 241-247, 2009a.

FREITAS, F. C. L. et al. Manejo de plantas daninhas na cultura do feijão-caupi. In: CONGRESSO NACIONAL DE FEIJÃO-CAUPI, 2., 2009, Bélem. Anais... [recurso eletrônico] Belém: 2009b. 
FREITAS, F. C. L. et al. Seletividade de herbicidas para a cultura do feijão-caupi. In: CONGRESSO BRASILEIRO DA CIÊNCIA DAS PLANTAS DANINHAS, 27., 2010. Ribeirão Preto. Anais... Ribeirão Preto: SBCPD, 2010. p. $1748-1752$.

FONTES, J. R. A.; GONÇALVES, J. R. P.; MORAIS, R. R. Tolerância do feijão-caupi ao herbicida oxadiazon. Pesq. Agropec. Trop., v. 40, n. 1, p. 110-115, 2010.

FREIRE FILHO, F. R. et al. Feijão-caupi no Brasil: produção, melhoramento genético, avanços e desafios. Teresina: Embrapa Meio-Norte, 2011. 84 p.

LINHARES, C. M. S. et al. Seletividade dos herbicidas fomesafen e bentazon+imazamox para a cultura do feijãocaupi. In: Congresso Brasileiro de Feijão-Caupi, 3, 2013. Recife, Anais eletrônicos ... Recife: IPA, 2013. Disponível em: <http://www.conac2012.org/resumos/pdf/023a.pdf>. Acesso em: 20 jul. 2013.

MARQUES, L. J. P. et al. Dinâmica de populações e fitossociologia de plantas daninhas no cultivo do feijão-caupi e mandioca no sistema corte e queima com o uso de arado. Planta Daninha, v. 29, p. 981-989, 2011. (Número Especial)

MESQUITA, H. C. Seletividade e eficácia de herbicidas em cultivares de feijão-caupi (Vigna unguiculata (L.) Walp.). 2011. 52 f. Dissertação (Mestrado em Fitotecnia) - Universidade Federal Rural do Semi-Árido, Mossoró, 2011.
SANTOS, H. G. et al. Sistema brasileiro de classificação de solos. 2.ed. Rio de Janeiro: Embrapa Solos, 2006. 306 p.

RODRIGUES, B. N.; ALMEIDA, F. S. Guia de herbicidas. 6.ed. Londrina: Grafmarke, 2011. 639 p.

SILVA, A. A.; SILVA, J. F. (Ed.). Tópicos em manejo de plantas daninhas. Viçosa, MG: Universidade Federal de Viçosa, 2007. 367 p.

SILVA, J. F.; ALBERTINO, S. M. F. Manejo de plantas daninhas. In: ZILLI, J.E.; VILARINHO, A. A.; ALVES, J. M. A. A cultura do feijão-caupi na Amazônia brasileira. Boa Vista: Embrapa Roraima, 2009. p. 223-243.

SILVA, J. B. F. et al. Controle de plantas daninhas em feijãode-corda em sistema de semeadura direta. Planta Daninha, v. 21, n. 1, p. 151-157, 2003.

SOCIEDADE BRASILEIRA DA CIÊNCIA DAS PLANTAS DANINHAS - SBCPD. Procedimentos para instalação, avaliação e análise de experimentos com herbicidas. Londrina: 1995. 42 p.

SOUZA, R. T. et al. Seletividade de combinações de herbicidas latifolicidas com lactofen para a cultura da soja. Sci. Agríc., v. 59, n. 1, p. 99-106, 2002.

VILARINHO, A. A. BRS Guariba - cultivar de feijão-caupi de alto desempenho em Roraima. 2007. Artigo em Hipertexto. Disponível em: <http://www.infobibos.com/ Artigos/2007_4/Guariba/index.htm>. Acesso em: 15 dez. 2011. 